\title{
Analytical, numerical and experimental study on capillary flow in a microchannel traversing a backward facing step
}

\author{
Ahmed Taher ${ }^{\mathrm{a}, \mathrm{b}, *}$, Benjamin Jones $^{\mathrm{b}}$, Paolo Fiorini ${ }^{\mathrm{b}}$, Liesbet Lagae ${ }^{\mathrm{a}, \mathrm{b}}$ \\ a Department of Physics, KU Leuven, Leuven, Belgium \\ ${ }^{\mathrm{b}}$ Life Science and Technology Department, IMEC v.z.w., Leuven, Belgium
}

\section{A R T I C L E I N F O}

\section{Article history:}

Received 26 March 2018

Revised 7 June 2018

Accepted 20 June 2018

Available online 21 June 2018

\section{Keywords:}

Capillary microfluidics

Lab on a chip

Numerical simulation

Backward facing step

\begin{abstract}
A B S T R A C T
Capillary flow traversing a backward facing step (BFS) in a microchannel at low Capillary and Weber numbers is investigated in detail using analytical, numerical and experimental techniques. The BFS's under study included both open surface, where a free surface is formed at the top to the channel, and closed surface, where a lid with a different contact angle than the base material is used. An analytical model valid for both geometries was derived to determine the capillary pressure as a function of the liquidgas interface position as it traverses the BFS. The model was validated against two different numerical simulation techniques: (1) surface energy minimization of the meniscus shape and (2) CFD simulation using the volume of fluid method. Comparison between the simulations and analytically derived model for a range of aspect ratios (0.5-3) and contact angles of the base material $\left(60^{\circ}-80^{\circ}\right)$ revealed that the analytical model works best at high contact angles $\left(>70^{\circ}\right)$ and high aspect ratios $(>2)$.

Furthermore, an analytically derived geometric condition required for spontaneous capillary flow over a BFS was developed. To validate the flow condition, experimental measurements were performed on microchannels with BFSs fabricated in silicon using deep reactive ion etching with aspect ratios ranging from 1.5 to 3.8. The contact angle of surfactant/water solutions ranged from $50^{\circ}$ to $85^{\circ}$ on the silanetreated silicon surfaces and from $93^{\circ}$ to $100^{\circ}$ on the PDMS top surface for the closed structure experiments. The experimental results were in good agreement with the analytically derived condition. The developed model is an enabling tool for designers of capillary-driven microfluidic systems.
\end{abstract}

(C) 2018 Elsevier Ltd. All rights reserved.

\section{Introduction}

In recent years, portable point-of-care devices have received increasing interest. Microfluidic systems are attractive for such applications due to their ability to handle small sample volumes, low reagent usage, and reduced waste (Chin et al., 2012). Commonly, external pumps, flow sensors and movable valves are used to perform liquid handling in microfluidic devices. Such components are relatively bulky, expensive, and difficult to integrate into miniaturized platforms (Junker et al., 2002). Capillary-based microfluidic devices provide an attractive solution for some applications since external equipment or movable parts are not needed for fluidic manipulation (Geravis and Delamarche, 2009). However, predicting and controlling capillary flow behavior in many structures of interest remains challenging.

\footnotetext{
* Corresponding author at: Life Science and Technology Department, IMEC v.z.w., Leuven, Belgium.

E-mail address: ahmed.taher@imec.be (A. Taher).
}

The capillary trigger valve, first described by Melin et al. (2003), has proven to be useful circuit element to realize some degree of flow control in a capillary-driven system. The capillary trigger valve utilizes a structure that is difficult to wet, usually a sudden geometric expansion, in order to stop the progression of a liquid-vapor interface in a channel. A second channel, usually at a right angle to the direction of propagation of the stopped fluid, is used to convey another fluid stream that meets with the stopped liquid-vapor interface to trigger or re-start the flow of the stopped fluid. Several different designs and methods of fabrication of capillary trigger valves have been proposed over the past several years (Zimmermann et al., 2008; Hitzbleck et al., 2012; Safavieh and Juncker, 2013; Majeed et al., 2015). Most recently, reliable capillary trigger valves have been reported using a two-depth structure and a hydrophobic cover (Safavieh and Juncker, 2013; Majeed et al., 2015). As discussed by Zhang et al. (2018), there are advantages to having a shallow trigger channel with a locally deep region only at the valve section; in this situation, the liquid-vapor interface in the trigger channel has to traverse a backward facing step (BFS) in order to trigger the valve. In some cases, the flow may stop at the $\mathrm{BFS}$, resulting in a failure to trigger the valve when intended. 
Abrupt changes in the geometric profile of a microchannel have been reported for various purposes in the literature. For instance, Chibbaro et al. (2009) used a square obstacle to stop the capillary filling of a microchannel at high contact angles $\left(<60^{\circ}\right)$. It was found in their study that the liquid front stops at the end of the obstacle which forms a BFS. Furthermore, patterning of a microchannel's surface in the form of stripes perpendicular to the advancement direction of the liquid-air meniscus, referred to as phaseguides, was reported as a means of capillary flow control (Vulto et al., 2011; Yildirim et al., 2014). The dead angle phaseguides that were used to stop the capillary flow propagation were in the form of BFSs. Hence, better understanding and modeling the behavior of capillary flow over a BFS is relevant to many applications.

Design and development of capillary-driven devices remains an expensive endeavor. Computational fluid dynamics (CFD) simulations using the volume of fluid (VOF) method carry a high computational cost. Prototyping also presents challenges and can be time consuming and costly especially for silicon-based processing. Better analytical tools to predict the performance characteristics of key components in a capillary system are needed. Exact solutions to the capillary pressure in surface tension dominated flows are scarce even for geometries of moderate complexity. However, approximate models have been proposed for several geometries of interest. Using surface energy based modeling approaches has yielded models of capillary pressure as a liquid-vapor interface traverses capillary valves of different geometries (Man et al., 1998; Leu and Chang, 2004; Cho et al., 2007; Chen et al., 2008; Yildirim, 2017; Gliere and Delattre, 2006; Berthier et al., 2007). For instance, Man et al. (1998) calculated the burst pressure of an electrically actuated capillary stop valve using a 2D surface energy model. A similar study was extended to a 3D meniscus model by Leu and Chang (2004) to determine the pressure barrier of a capillary stop valve for a centrifugal sample separator. However, the important geometry of a BFS in a capillary system is less well studied. Here, we develop an analytical model to determine the capillary pressure for different positions of the liquid-gas interface with respect to a BFS in a microchannel as a function of the channel geometry and the surface tension coefficient using surface energy calculations. The developed model is used to obtain a geometric condition which needs to be satisfied in order to allow spontaneous capillary flow to traverse a BFS.

The capillary pressure as a function of interface position generated by the analytical model are validated against simulation results from two different numerical simulation techniques. The first simulation technique is performed by the Surface Evolver software (Brakke, 1992), a free tool to determine the liquid-gas interface shape using surface energy minimization. Furthermore, the VOF method is employed using the commercially available CFD software ANSYS Fluent. Numerical simulations are conducted in three different aspect ratios ranging from 0.5 to 3 (height/width) with three different contact angles ranging from $60^{\circ}$ to $80^{\circ}$. The spontaneous flow condition is validated against experimental results from testing open (without a top cover) and closed (with a PDMS cover) microchannels with BFSs fabricated in silicon using deep reactive ion etching (DRIE). The experiments are conducted for a range of microchannels aspect ratios and contact angles.

\section{Theory and analytical modeling}

\subsection{Surface energy modeling}

The surface tension coefficient, $\gamma$, is the force per unit length acting along the interface of immiscible phases due to the imbalance of inter-molecular forces across the interface. In a liquid-gassolid surface system, the molecules at the liquid-gas interface re- arrange themselves in order to minimize the surface energy, hence a meniscus is formed between the two phases. The surface tension coefficient for each pair of the three phases plays a role in shaping the liquid-gas meniscus. The capillary pressure is defined as the pressure difference on either sides of a liquid-gas meniscus $\left(P_{\text {gas }}-P_{\text {liquid }}\right)$. The value of the capillary pressure determines the dynamics of the spontaneous liquid flow inside a microchannel: either the liquid advances (positive capillary pressure), retracts (negative capillary pressure) or remains stationary (zero capillary pressure).

The equations for interfacial equilibrium can be derived from the energy minimization considerations. The surface energy ( $E$ ) of a liquid droplet having an occupancy volume $(V)$ inside a microchannel can be calculated by (Chakraborty and Chakraborty, 2010)

$E=\sum_{i \neq j} A_{i j} \gamma_{i j}$

where $A_{i j}$ is the interfacial area between the phases $i$ and $j$ with a corresponding surface tension coefficient $\gamma_{i j}$. Note that $i, j=$ $s, l$, and $g$ for a three-phase system consisting of solid, liquid, and gas phases, respectively.

The capillary pressure jump $\left(\Delta P_{c}\right)$ across the interface can be defined as

$\Delta P_{c}=\frac{\partial E}{\partial V}$

The geometry of interest is shown in Fig. 1. As will be discussed in the following sections, Eqs. (1) and (2) can be applied to a model of the liquid-gas-solid surface system to determine the capillary pressure as the liquid-gas interface traverses the backward facing step.

The proceeding analysis is performed under the assumption that surface tension forces dominate over inertial and viscous forces. This assumption implies that the interface shape is determined only by the surface tension effects and the effects of inertia are negligible. The relation between surface tension forces and inertial forces can be expressed in terms of Weber numbers, $W e=\rho v^{2} l / \gamma$, and the one between surface tension forces and viscous forces can be expressed in terms of capillary numbers, $C a=\mu \nu / \gamma$. For flow in a microchannel, the inertial and viscous effects on the interface shape can be considered negligible as long as $W e<0.015$ and $C a<0.02$ (Hoffman, 1983). However, this is not seen as a major limitation of the foregoing analysis as many capillary-driven flows of interest meet such conditions. Furthermore, the proceeding analysis is only valid for contact angles higher than $45^{\circ}$. For lower contact angles, spontaneous corner flow of the liquid may precede the bulk of the liquid in the microchannel, based on Concus-Finn condition (Weislogel and Lichter, 1998), which will result in inconsistencies in the developed model.

In the following sections, Eqs. (1) and (2) are applied to the liquid-gas interface at different locations of a microchannel with a BFS. First, the equations are applied to the liquid-gas interface at the shallow part of the microchannel before the step. Second, the liquid-gas interface surface energy is analyzed when the interface is pinned to the edge of the step. Last, the analysis is done to the interface when the liquid starts wetting the vertical wall after the step edge.

\subsection{Stage I - Liquid-gas interface in the microchannel}

When the liquid-gas interface is located before the step (i.e., $x<x_{1}$ ), as shown in Fig. 2, the meniscus meets the solid walls of the channel at an angle equal to the contact angle as a characteristic angle of the solid, liquid and gas phases of the capillary system. In the context of this research, we assume that the side walls 


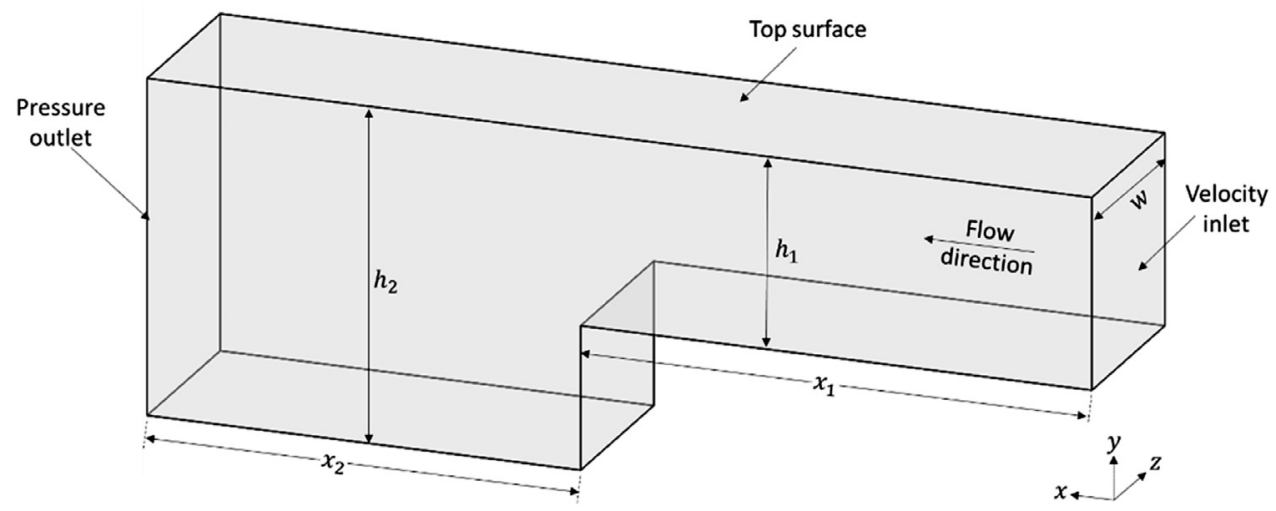

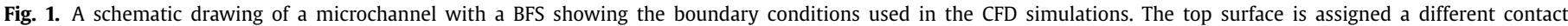
angle than the other wetted walls in the developed models.

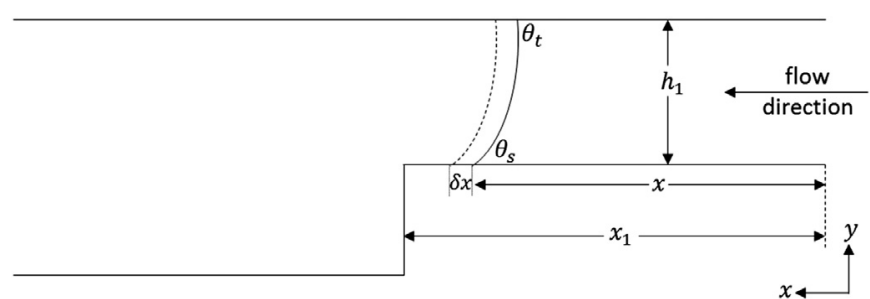

Fig. 2. Schematic of backward facing step with the meniscus positioned before reaching the step.

and the bottom surface have the same contact angle $\left(\theta_{s}\right)$, while the contact angle of the top surface is $\theta_{t}$. The capillary pressure can be calculated from the Young-Laplace equation or, alternatively, may also be derived from the surface energy analysis, as following.

From Eq. (1), the surface energy of the liquid in the microchannel can be evaluated:

$E=A_{l s, s} \gamma_{l s, s}+A_{s v, s} \gamma_{s v, s}+A_{l s, t} \gamma_{l s, t}+A_{s v, t} \gamma_{s v, t}-A_{l v} \gamma_{l v}$

where $A_{l s, s}, A_{s v, s}, A_{l s, t}, A_{s v, t}$ and $A_{l v}$ are the interfacial areas between the liquid-solid (side and bottom surfaces), solid-gas (side and bottom surfaces), liquid-solid (top surface), solid-gas (top surface) and liquid-gas phases, respectively, and $\gamma_{l s, s}, \gamma_{s v, s}, \gamma_{l s, t}$, $\gamma_{s v, t}$ and $\gamma_{l v}$ are the corresponding surface tension coefficient between each pair. Inserting Eq. (3) into Eq. (2) and applying the chain rule yields

$$
\begin{aligned}
\Delta P_{c}= & \frac{\partial E}{\partial x} \frac{\partial x}{\partial V}=\left(\gamma_{l s, s} \frac{\partial A_{l s, s}}{\partial x}+\gamma_{s v, s} \frac{\partial A_{s v, s}}{\partial x}+\gamma_{l s, t} \frac{\partial A_{l s, t}}{\partial x}\right. \\
& \left.+\gamma_{s v, t} \frac{\partial A_{s v, t}}{\partial x}-\gamma_{l v} \frac{\partial A_{l v}}{\partial x}\right) \cdot \frac{\partial x}{\partial V}
\end{aligned}
$$

By analyzing the geometry in Fig. 2, it can be deduced that $\partial A_{l s, s} / \partial x=-\partial A_{s v, s} / \partial x=2\left(h_{1}+w\right), \quad \partial A_{l s, t} / \partial x=-\partial A_{s v, t} / \partial x=$ $w, \partial A_{l v} / \partial x=0$, and $\partial x / \partial V=1 / h_{1} w$. Note that $x$ is the only independent variable for this geometry. The contact angle is defined by the mechanical equilibrium of a liquid drop under the action of three interfacial tensions (Yuan and Lee, 2013)

$\gamma_{l v} \cos \theta_{s}=\gamma_{l s, s}-\gamma_{s v, s}$

$\gamma_{l v} \cos \theta_{t}=\gamma_{l s, t}-\gamma_{s v, t}$

Then from Eqs. (4) and (5), an expression for the capillary pressure can be derived

$\Delta P_{c}=\gamma_{l v}\left(\frac{2 \cos \theta_{s}}{w}+\frac{\cos \theta_{s}+\cos \theta_{t}}{h_{1}}\right)$

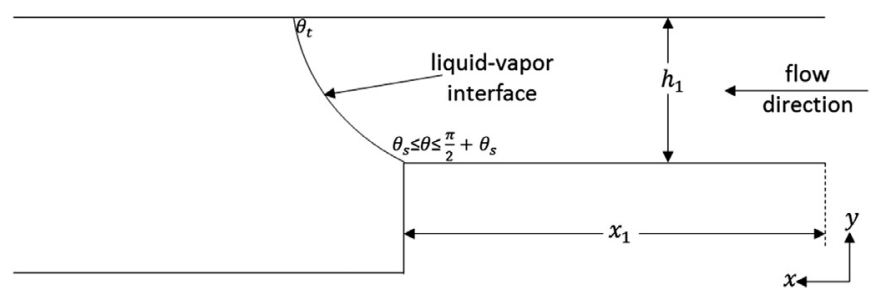

Fig. 3. Schematic of backward facing step with the meniscus pinned at the step

The last equation can be confirmed by the well-known YoungLaplace equation. However, for the next two stages of the liquidgas interface traversing a BFS, presented in the following two sections, the capillary pressure formula can more easily be derived from surface energy analysis.

\subsection{Stage II - Liquid-gas interface pinned at the step edge}

In this Section 2.3 and the following (2.4), geometrical simplifications to the interface shape are made for analytical convenience. Calculations of the interfacial areas and the liquid volume were done based on the assumption of a 2D curved liquid-gas interface (i.e., assuming the position of the liquid-gas interface is only a function of $\mathrm{x}$ and $\mathrm{y}$ coordinates and not the $\mathrm{z}$ coordinate). The implications this has on the results will be discussed later in this manuscript.

Once the liquid-gas interface reaches the step $\left(x=x_{1}\right)$, the interface is pinned to the step edge until the angle between the liquid-gas interface and the bottom wall of the channel is equal to $\pi / 2+\theta_{s}$, as shown in Fig. 3. Consequently, the angle between the liquid-gas interface and the bottom surface of the microchannel changes to accommodate the change in the interface curvature while the angles between the interface and the side and top surfaces of the microchannel remain constant and equal to the characteristic contact angles $\theta_{s}$ and $\theta_{t}$, respectively.

In this case, the $x$-position of the liquid-gas interface stays constant while the angle $(\theta)$ between the interface and the bottom surface of the channel changes from $\theta_{s}$ to $\pi / 2+\theta_{s}$. Thus, the only independent variable in this case is $\theta$. This leads to a capillary pressure equation as follows

$\Delta P_{c}=\gamma_{l v}\left(-\frac{\partial A_{l v}}{\partial \theta}+\cos \theta_{s} \frac{\partial A_{l s, s}}{\partial \theta}+\cos \theta_{t} \frac{\partial A_{l s, t}}{\partial \theta}\right) \cdot \frac{\partial \theta}{\partial V}$

where

$\frac{\partial A_{l v}}{\partial \theta}=-w h_{1}\left(\frac{1}{\cos \theta+\cos \theta_{t}}-\frac{\left(\theta+\theta_{t}-\pi\right) \sin \theta}{\left(\cos \theta+\cos \theta_{t}\right)^{2}}\right)$ 


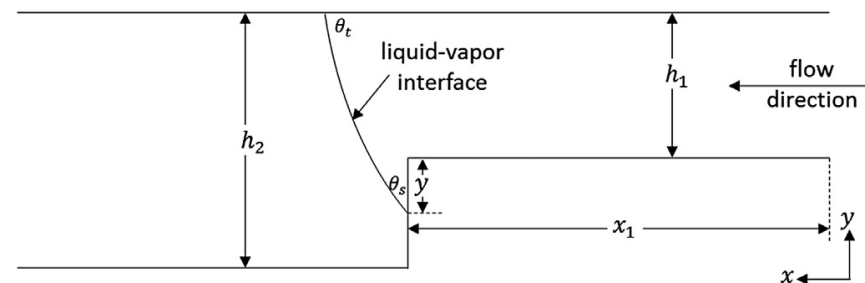

Fig. 4. Schematic of backward facing step with the meniscus wetting the vertical wall after the step.

$$
\begin{aligned}
\frac{\partial A_{l s, s}}{\partial \theta}= & \frac{2 h_{1}^{2}}{\left(\cos \theta+\cos \theta_{t}\right)^{2}}\left[1+\cos \left(\theta+\theta_{t}\right)\right. \\
& \left.+\frac{\sin \theta\left(\theta+\theta_{t}-\pi+\sin \left(\theta+\theta_{t}\right)\right)}{\cos \theta+\cos \theta_{t}}\right]
\end{aligned}
$$

$\frac{\partial A_{l s, t}}{\partial \theta}=\frac{w h_{1}\left(1+\cos \left(\theta+\theta_{t}\right)\right)}{\left(\cos \theta+\cos \theta_{t}\right)^{2}}$

and

$$
\begin{aligned}
\frac{\partial \theta}{\partial V}= & {\left[\frac { w h _ { 1 } { } ^ { 2 } } { ( \operatorname { c o s } \theta + \operatorname { c o s } \theta _ { t } ) ^ { 2 } } \left(1+\cos \left(\theta+\theta_{t}\right)\right.\right.} \\
& \left.\left.+\frac{\sin \theta\left(\theta+\theta_{t}-\pi+\sin \left(\theta+\theta_{t}\right)\right)}{\cos \theta+\cos \theta_{t}}\right)\right]^{-1}
\end{aligned}
$$

\subsection{Stage III - Liquid-gas interface wetting vertical wall of the step}

After the liquid-gas interface is released from the step, the liquid starts wetting the vertical wall of the step, as shown in Fig. 4. As long as the interface does not reach the bottom surface of the channel after the step $\left(0<y<h_{2}-h_{1}\right)$, the $x$-position of the interface remains constant as well as the angle between the interface and the vertical wall (the contact angle, $\theta_{s}$ ). Therefore, the only independent variable in this case is the vertical position $(y)$.

Then, the capillary pressure can be determined as

$\Delta P_{c}=\gamma_{l v}\left(-\frac{\partial A_{l v}}{\partial y}+\cos \theta_{s} \frac{\partial A_{l s, s}}{\partial y}+\cos \theta_{t} \frac{\partial A_{l s, t}}{\partial y}\right) \cdot \frac{\partial y}{\partial V}$

where

$\frac{\partial A_{l v}}{\partial y}=w\left(\frac{\theta_{s}+\theta_{t}-\pi / 2}{\sin \theta_{s}-\cos \theta_{t}}\right)$

$$
\begin{aligned}
\frac{\partial A_{l s, s}}{\partial y}= & w+2\left(h_{1}+y\right)\left(\frac{\sin \theta_{t}-\cos \theta_{s}}{\sin \theta_{s}-\cos \theta_{t}}\right. \\
& \left.+\frac{\theta_{s}+\theta_{t}-\pi / 2+\cos \left(\theta_{s}+\theta_{t}\right)}{\left(\sin \theta_{s}-\cos \theta_{t}\right)^{2}}\right)
\end{aligned}
$$

$\frac{\partial A_{l s, t}}{\partial y}=\frac{w\left(\sin \theta_{t}-\cos \theta_{s}\right)}{\sin \theta_{s}-\cos \theta_{t}}$

and

$\frac{\partial y}{\partial V}=\left[w\left(h_{1}+y\right)\left(\frac{\sin \theta_{t}-\cos \theta_{s}}{\sin \theta_{s}-\cos \theta_{t}}+\frac{\theta_{s}+\theta_{t}-\pi / 2+\cos \left(\theta_{s}+\theta_{t}\right)}{\left(\sin \theta_{s}-\cos \theta_{t}\right)^{2}}\right)\right]^{-1}$

\subsection{The special case of an open (free) surface microchannel}

Certain applications require spotting chemical reagents inside the microfluidic device to store them in the dry form for better chemical stability. Thus, for such applications, it is more convenient to use open surface microfluidic structures so that the dried reagents can be directly spotted inside the structure after fabrication (Zimmermann et al., 2005). Capillary flow in a microchannel with an open top surface can be considered by setting the top contact angle $\theta_{t}=180^{\circ}$.

Fig. 5 shows the capillary pressure values for different contact angles of the top surface of a microchannel with a BFS for the two last stages: $(A)$ when the interface is pinned to the step edge with an angle, $\theta$, and (B) when the liquid starts wetting the vertical wall. The microchannel used in this example has a width of $40 \mu \mathrm{m}$, a $60 \mu \mathrm{m}$ height before step and an $80 \mu \mathrm{m}$ height after the step. It can be found from the figure that open surface $\left(\theta_{t}=180^{\circ}\right)$ microchannels with a BFS will always have the lowest capillary pressure for a given interface angle $\theta$ or vertical distance $y$. The validation of the model in the next sections focuses on BFS's in open surface microchannels.

\subsection{Spontaneous flow condition}

In order for the liquid to flow spontaneously through a microchannel with a BFS, the capillary pressure jump across the liquid-gas interface needs to be positive at all times. By differentiating equations 6 with respect to the independent variable, $x$, it can be found that before the step the capillary pressure is constant or $d P_{c} / d x=0$. However, when the liquid-gas interface is pinned to the step, the rate of change of the capillary pressure is always negative for $\theta_{s}>\theta>\pi / 2+\theta_{s}$ (i.e., $d P_{c} / d \theta<0$ ). Moreover, the capillary pressure derivative after the step with respect to the vertical position, $y$, is always positive for $0<y<h_{2}-h_{1}$ (i.e., $d P_{c} / d y>0$ ). As a result, the minimum value of the capillary pressure occurs when the liquid-gas interface is at the end of the pinning stage and starts to wet the vertical wall after the step, which is either when $\theta=\pi / 2+\theta_{s}$ at Eq. (7) or when $y=0$ at Eq. (12). To ensure a positive value of the capillary pressure at this position, the ratio between the microchannel's height to width has to fulfill the following condition.

$\frac{h_{1}}{w}>\frac{\sin \theta_{s}-\cos \theta_{t}}{2 \cos \theta_{s}}$

By considering $\theta_{t}=180^{\circ}$, for an open surface microchannel, the spontaneous liquid flow condition can be represented as

$\frac{h_{1}}{w}>\frac{\sin \theta_{s}+1}{2 \cos \theta_{s}}$

Although the model presented in this section is applicable only for a right angle BFS in a microchannel, it can be extended easily to other geometries where no change in the geometry happens in the third dimension. For these cases, 2D surface energy modeling can be used to estimate the capillary pressure as a function of the liquid-gas interface location. However, for other cases where the change in the geometry happens in the three dimensions, such as phaseguides with tilted steps, a more complex 3D model is required or numerical simulation can be conducted for each case.

In the proceeding sections, the capillary pressure values estimated by analytical model is compared to numerical simulation data. Then, the spontaneous flow condition is validated against experimental data for open and closed surface microchannels.

\section{Analytical model validation against numerical simulation}

In order to validate the analytical model, we conducted numerical simulations of capillary flow over BFS problem using two dif- 


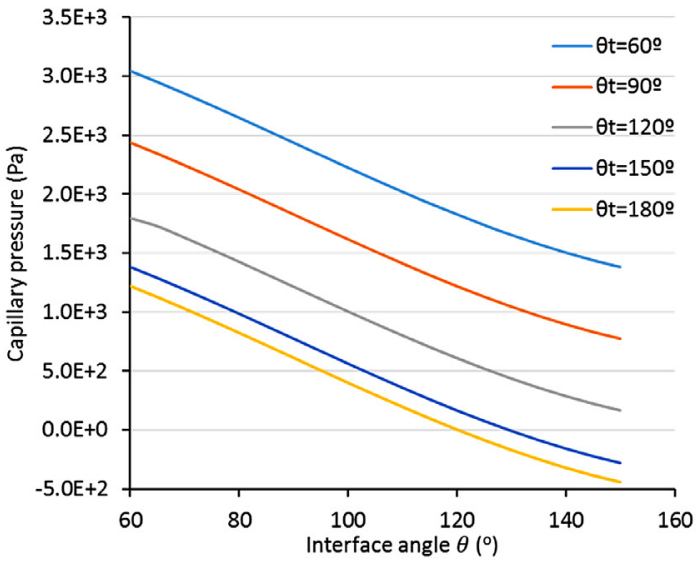

(A)

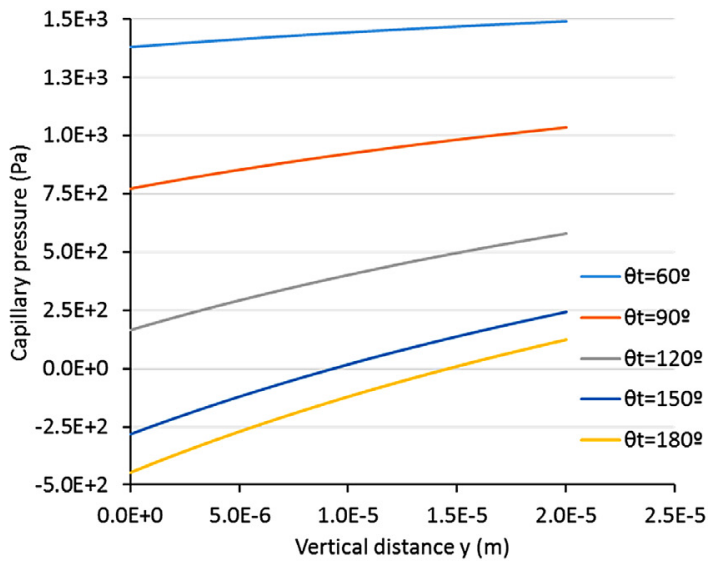

(B)

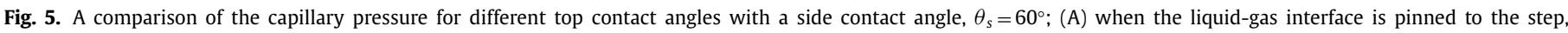
and (B) when it is wetting the vertical wall.

ferent simulation techniques. The first one is a surface energy minimization approach using Surface Evolver (Brakke, 1992). The second approach is computational fluid dynamics (CFD) simulations of two-phase flow by ANSYS Fluent software implementing the volume of fluid approach (Hirt and Nichols, 1981). Comparing both approaches to the analytical model imparts more confidence to the outcomes of the numerical analysis.

\subsection{Surface evolver}

The Surface Evolver software evolves the surface towards minimal energy by a gradient descent method (Brakke, 1992). The surface energy minimization approach represents a powerful simulation tool for equilibrium surface shapes of liquids subject to surface tensions and other energies under various constraints (Yuan and Lee, 2013; Zimmermann et al., 2005). Therefore, flow field calculations are not included in the surface energy simulation. However, this is not considered as a limitation in our case as long as capillary number, $\mathrm{Ca}$, and Weber number, We, are small enough so that fluid dynamics effects on the interface shape are negligible (Hoffman, 1983). The Surface Evolver model was constructed by defining level set constraints to an initial meniscus surface in contact with the solid surfaces such as fixed liquid volume constraint and fixed contact angle between the liquid-gas free surface and the solid walls for the geometry shown in Fig. 1. The final free surface shape was obtained when the energy function reaches a minimal value.

The meniscus surface mesh was refined repetitively to ensure sufficient resolution to the surface curvature. A refinement criterion was used so that the number of mesh elements depends on the surface area of the meniscus interface. The number of mesh facets in the simulations range from 256 to 2000 facets. A convergence criterion was set by comparing the relative value of change in capillary pressure after each 100 iterations to a fixed value. The value of the convergence criterion was determined by validating the model to the well-established analytical model of the capillary flow in a microchannel with open surface (Eq. 6). We chose $10^{-5}$ as value of change in capillary pressure after 100 iterations to be our convergence criterion for all the Surface Evolver simulations conducted in this study.

\subsection{CFD simulation}

The volume of fluid (VOF) method (Hirt and Nichols, 1981) was used to model two-phase flow of immiscible phases. The sur- face tension forces along the liquid-gas interface are included as a source term to the Navier-Stokes equation. The capillary pressure is determined by calculating the meniscus surface curvature from local gradients in the surface normal to the interface (Saha and Mitra, 2009). However, the exact location of the liquid-gas interface is difficult to determine without significant numerical cost because the VOF model does not resolve the interface location with subgrid resolution (Hoang et al., 2013).

A liquid drop of fixed volume was modeled in the numerical simulation by initializing the liquid volume (by setting the volume fraction to 1 ) in the microchannel and setting the liquid velocity to zero at the velocity inlet boundary condition (see Fig. 1). The capillary pressure was computed as the pressure difference between the inlet and outlet of the microchannel, which, for a stagnant fluid, equals the pressure jump across the liquid-vapor interface. The convergence of the solution was considered by iteration until a constant value of the capillary pressure was reached in the transient solution. In contrast to the Surface Evolver software, which is used only to model static liquid volumes at equilibrium, VOF method can be used to model the surface tension forces for liquids in motion. However, in the scope of this study, we use both methods to study the capillary pressure of fixed liquid volumes at equilibrium.

\subsection{Results comparison}

As mentioned earlier, the comparisons between numerical simulation and the analytical model will focus on open surface microchannels. Three different aspect ratios, which can be defined as channel height divided by width before the step, were studied, as shown in Table 1 . For all geometries, the step is at $x=200 \mu \mathrm{m}$ from the inlet and the channel depth after the step $\left(h_{2}\right)$ is $150 \mu \mathrm{m}$. Three contact angles of the side walls and the bottom surface $\left(\theta_{s}\right)$, $60^{\circ}, 70^{\circ}$ and $80^{\circ}$, were investigated using the first geometry while $\theta_{s}$ was set to $60^{\circ}$ for the other geometries. The value of surface tension coefficient, $\gamma_{l v}$, used in all simulations is $0.073 \mathrm{~N} / \mathrm{m}$.

Fig. 6 shows the comparison between the analytical model, the Surface Evolver model and the CFD simulation for the first geometry with a contact angle of $60^{\circ}$. The capillary pressure is constant in the microchannel before reaching the step, as expected for a constant cross section channel. When the liquid-gas interface arrives at the step, the bottom of the interface becomes pinned to the step until the angle between the interface and the bottom of the channel equals $\pi / 2+\theta_{s}$. The capillary pressure value decreases gradually until it reaches its minimum value just before the inter- 
Table 1

\begin{tabular}{lllll}
\multicolumn{2}{l}{ List of geometrical parameters under study. } \\
\hline Geometry & Channel width $(w)(\mu \mathrm{m})$ & Channel height $\left(h_{1}\right)(\mu \mathrm{m})$ & Aspect ratio $\left(h_{1} / w\right)$ & Contact angles $\left(\theta_{s}^{\circ}\right)$ \\
\hline 1 & 40 & 60 & 1.5 & $60,70,80$ \\
2 & 20 & 60 & 3 & 60 \\
3 & 40 & 20 & 0.5 & 60 \\
\hline
\end{tabular}

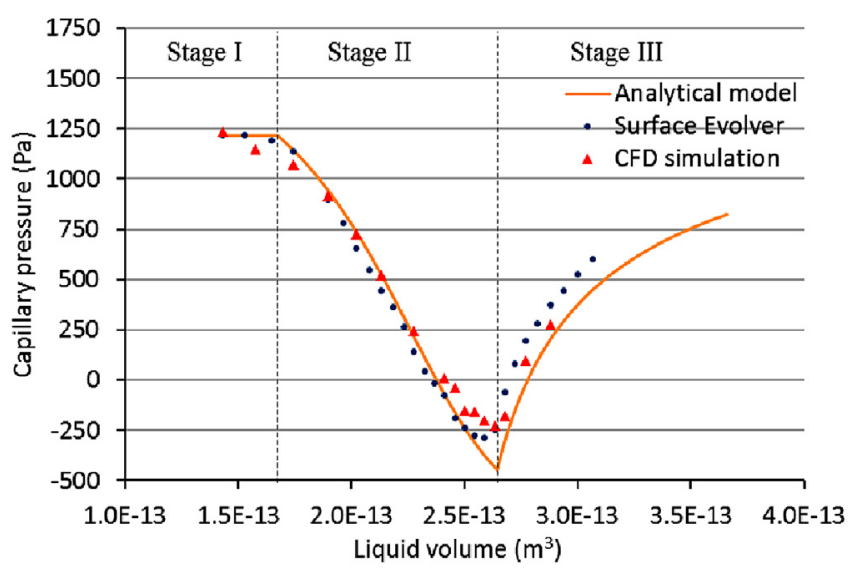

Fig. 6. Capillary pressure vs. liquid volume in an open surface BFS for Geometry 1 with a side contact angle, $\theta_{s}$, of $60^{\circ}$.

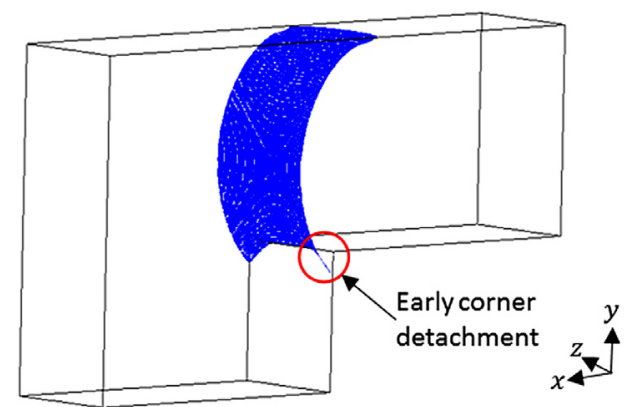

Fig. 7. Liquid-gas interface for the first geometry with side contact angle of $60^{\circ}$ using CFD simulation. Note the corners at the bottom of the liquid-gas interface have started to wet the vertical wall whilst the center of the interface remains pinned.

face leaves the step and starts wetting the vertical wall. This gradual decrease in the capillary pressure happens because the increase in the liquid-gas surface energy is more than the increase in liquidsolid surface energy as the liquid volume increases.

It can be found from the figure that both the Surface Evolver modeling and the CFD simulation are in good agreement with the analytical model. However, Surface Evolver and CFD simulation data points tend towards a shift to the left of the analytical model curve. This is because the analytical model assumes 2D curvature of the meniscus surface in the $x-y$ plane only and does not take into account any change in interface shape in the $z$ direction. Thus the liquid volume calculated using the analytical model is more than its actual value determined by Surface Evolver or CFD simulation at each moment. Also, the minimum value of the capillary pressure in the simulations is more than the minimum value in the analytical model calculations due to the manner of pinning and detachment of the liquid-gas interface from the step to wet the vertical wall after the step. The analytical model assumes that the interface is detached from the step at once as it transitions from Stage II to Stage III, while the numerical simulation indicates that the pinning and detachment process happens gradually starting from the corners of the microchannels, as shown in Fig. 7. Due to this gradual detachment, the minimum value of the capillary pressure across the interface in the numerical simulations does not reach as low of a value as in the analytical model.

\subsection{Effect of the contact angle}

Fig. 8 shows a comparison between the analytical model and the Surface Evolver modeling for different contact angles of the side and bottom walls $\left(\theta_{s}\right)$. It is found that higher contact angles yield better agreement between the analytical model and the Surface Evolver results. The reason for this is that at higher contact angles the liquid-gas interface is less curved in z-direction and the 2D simplification of the meniscus shape in analytical model is a better approximation of the actual interface shape.

\subsection{Aspect ratio effect}

The comparison between the Surface Evolver model and the analytical model for different aspect ratios can be found in Fig. 9. The capillary pressure vs. liquid volume in the microchannel for the three geometries (Table 1) was calculated using a contact angle of $60^{\circ}$. Higher aspect ratios yield better agreement between the Surface Evolver model and the analytical model, especially near the minimum value of the curve where the liquid-gas interface becomes unpinned from the edge of the step. Since the sidewall effect is more dominant at higher aspect ratios, the effect of the corners on detachment of the meniscus from the step edge (see Fig. 7) is less prominent. Hence, the difference in capillary pressure versus liquid volume between the analytical model, which neglects such effect, and the Surface Evolver model is less distinct at higher aspect ratios.

It can be deduced from the last section that the analytical model works best at high contact angles $\left(\theta_{\mathrm{s}}>70^{\circ}\right)$ and high aspect ratios $\left(h_{1} / w>2\right)$. However, it still gives acceptable results at medium values of contact angles and aspect ratios. In the following section, the spontaneous flow condition is validated against experimental data for different aspect ratios and contact angles of the top, side and bottom surfaces.

\section{Experimental validation of the spontaneous flow condition}

To confirm the spontaneous flow condition, developed from the analytical model, multiple structures were tested to check whether the liquid will flow spontaneously over a BFS in a microchannel. Five aspect ratios $\left(h_{1} / w\right)$ were used in the testing ranging from 1.5 to 4 . Furthermore, various contact angles were tested ranging from $90^{\circ}$ to $180^{\circ}$ for the top surface and from $50^{\circ}$ to $85^{\circ}$ for the side walls and the bottom surface. This section explains the experimental setup used for characterization and discusses the results of the experimental validation.

\subsection{Experimental setup}

The test devices were fabricated in silicon using deep reactive ion etching (DRIE). The microchannels were etched in two depths; the deep one is approximately $160 \mu \mathrm{m}$ and the shallow depth is approximately $40 \mu \mathrm{m}$ (actual measured dimensions are shown in Table 2). The microchannels surface was then covered 


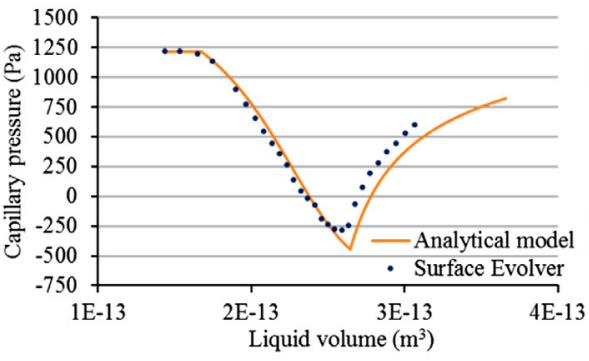

(A)

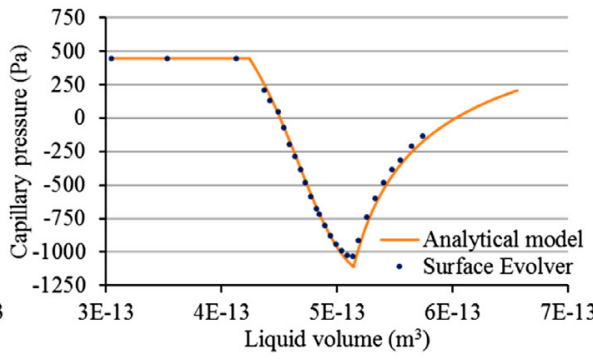

(B)

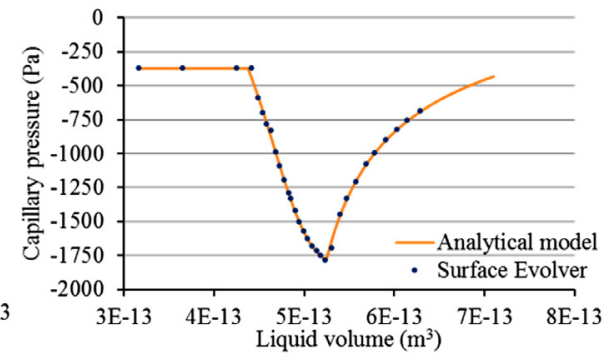

(C)

Fig. 8. Capillary pressure vs. liquid volume in the microchannel for Geometry 1 with contact angle $(\mathrm{A}) \theta_{s}=60^{\circ},(\mathrm{B}) \theta_{s}=70^{\circ}$ and $(\mathrm{C}) \theta_{s}=80^{\circ}$.

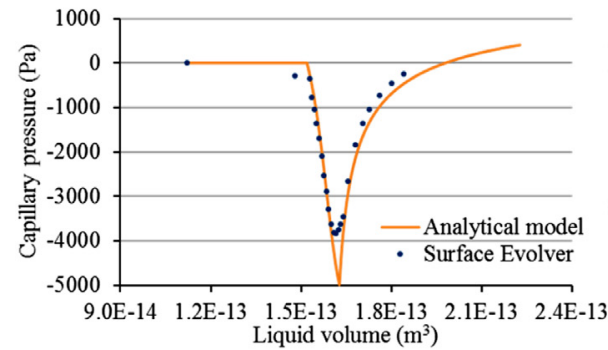

(A)

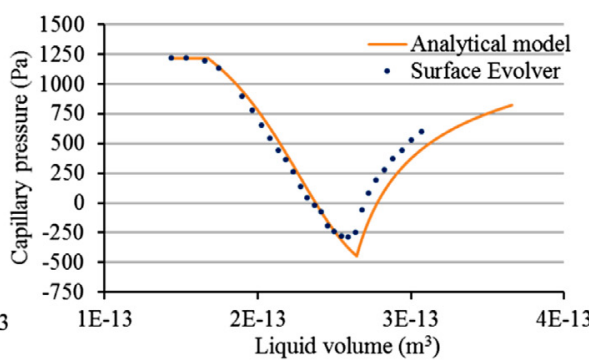

(B)

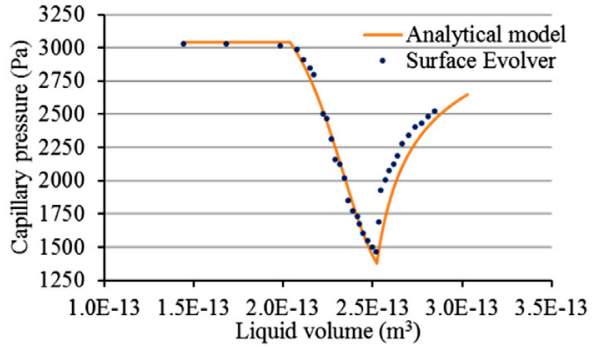

(C)

Fig. 9. Capillary pressure vs. liquid volume for (A) Geometry $3\left(h_{1} / w=0.5\right)$, (B) Geometry $1\left(h_{1} / w=1.5\right)$ and (C) Geometry $2\left(h_{1} / w=3\right)$ with contact angle $\theta_{s}=60^{\circ}$.

Table 2

Dimensions of the microchannels used in the experiments.

\begin{tabular}{llll}
\hline Microchannel & $\mathrm{w}(\mu \mathrm{m})$ & $\mathrm{h}_{1}(\mu \mathrm{m})$ & $\mathrm{h}_{1} / \mathrm{w}$ \\
\hline 1 & 10 & 38.3 & 3.83 \\
2 & 15 & 40 & 2.67 \\
3 & 20 & 41.6 & 2.08 \\
4 & 25 & 43.2 & 1.73 \\
5 & 30 & 44.7 & 1.49 \\
\hline
\end{tabular}

by a $200 \mathrm{~nm}$ layer of thermal oxide grown on the silicon surfaces. More details about the fabrication procedure can be found in Majeed et al. (2015). The test devices were fabricated as open surface microchannels. However, some test devices were covered by polydimethylsiloxane (PDMS) sheets to test microchannels with top surfaces, as well.

In order to control the contact angle between the liquidgas interface and the surface of the microchannels, we used 11azidoundecyltrimethoxysilane to render the silicon oxide surfaces hydrophobic using a vapor-phase deposition (Vos et al., 2018). The working fluid used in the experiments was water with a different amounts of Tween 20 surfactant added to obtain a range of contact angles. The static equilibrium contact angle for each Tween 20 concentration was measured using the Sessile Drop Method on the top surface of the test device. Fig. 10 shows the contact angles on the coated silicon surface corresponding to the different Tween 20 solutions. Regarding the top PDMS surface of the channels, the contact angles ranged from $93^{\circ}$ for working fluid with $0.005 \%$ concentration of Tween 20 surfactant to $100^{\circ}$ for $0.002 \%$ concentration of the surfactant.

It is worth noting that although the liquids are in motion during the experiments, the very low capillary numbers $\left(<10^{-4}\right)$ attained implies that the contact angle should be very close to the static advancing contact angle, which may differ a few degrees from the measured equilibrium contact angle. However, the difference in surface roughness between the flat top surface of the test device and the microchannel walls is expected to also have an

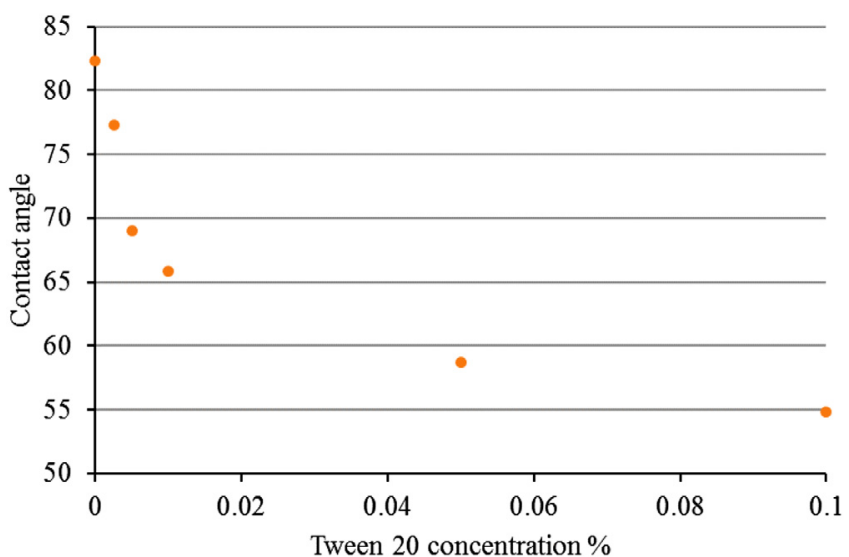

Fig. 10. The contact angle between the liquid-vapor interface and the silanized silicon oxide surfaces of the microchannel corresponding to different Tween 20 solution concentrations.

impact on the contact angle that is difficult to characterize experimentally. The more readily measurable equilibrium contact angles provide a convenient reference value to compare to the developed analytical models. But the effects mentioned may contribute to a small source of experimental error that should be considered when comparing results.

Five different microchannel geometries were used in the experimental testing. The width of channel ranges from 10 to $30 \mu \mathrm{m}$ which generates channel aspect ratio between the height and the width ranging from 1.5 to 3.8 with the shallow etch depth. Table 2 shows the dimensions of the microchannels used in the experiments.

\subsection{Results and discussion}

For microchannels with a top surface, low concentrations of the Tween 20 surfactant, ranging from $0.002 \%$ to $0.005 \%$, were used in the working fluid to generate relatively high contact angles that 


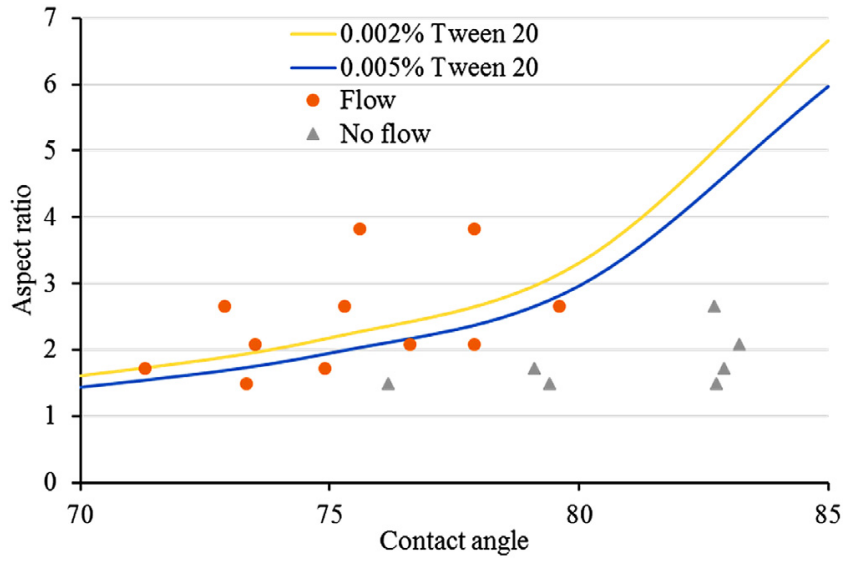

Fig. 11. Experimental results against the critical value of the aspect ratio from the spontaneous flow condition.

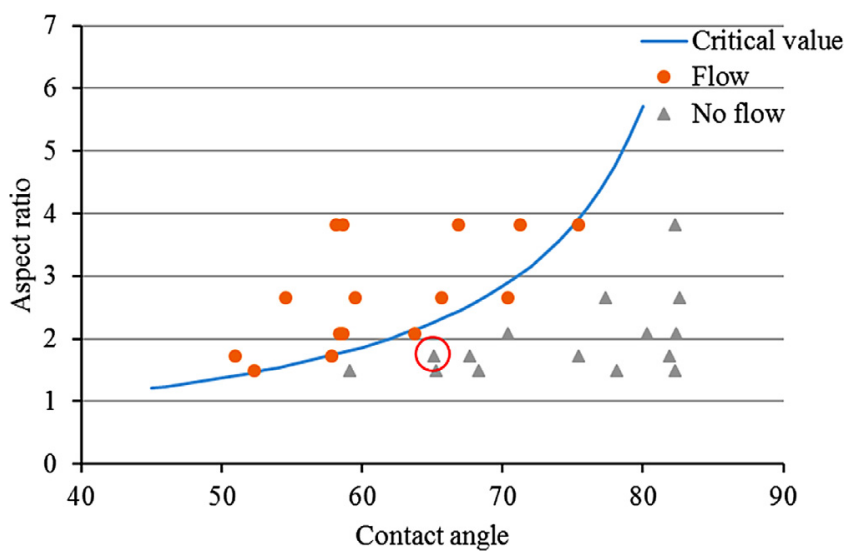

Fig. 12. Experimental results against the critical values from the spontaneous flow condition for open surface microchannels. For the circled point, the flow stopped for about $1 \mathrm{~min}$ before it started to flow again.

lie close to the critical values at which the liquid may or may not flow over the BFS. Fig. 11 shows the critical aspect ratio $\left(h_{1} / w\right)$ curves (solid lines) generated from the spontaneous flow condition (Eq. 20). As the contact angle of the top surface changes for each Tween 20 concentration, the critical value curve also shifts, as shown in the figure. The region below the critical value curve represents where the liquid flow is expected to stop in a BFS, whereas the region above the curve should flow.

It can be confirmed from Fig. 11 that the experimental data are in agreement with the analytically derived condition as most of the liquid-flow cases lie on top of the critical value curve and the noflow cases lie below it. However, it can be noticed from the figure as well, that there are some liquid-flow points that lie just under the critical value curve. There are a few possible explanations for this discrepancy. First, as mentioned previously, the surface roughness of the microchannel walls due to the DRIE fabrication process (Amin et al., 2012) may lead to lower contact angles inside the microchannel than the contact angles measured on planar, smooth surfaces (Kubiak et al., 2011). Second, as shown in Fig. 6, due to the assumption of 2D curvature of the liquid-gas interface, the actual minimum value of the capillary pressure is a little bit more than the determined value from the analytical model.

Similar to the flow in microchannels with top surfaces, Fig. 12 shows the experimental data for open surface microchannels $\left(\theta_{t}=\right.$ $180^{\circ}$ ). Again, the region above the critical value curve (Eq. 21) is expected to yield spontaneous flow across the BFS while below, the flow should stop. The experimental results remain in good agree- ment with the analytically developed condition, although some of the liquid-flow points lie just below the critical value curve due to the same reasons as discussed for closed surface microchannels. However, in the circled no-flow point in Fig. 12, the liquid flow stopped for about $1 \mathrm{~min}$ and then it started to flow again. Explanations for this nonadherent data point include possible misalignments between deep and shallow etches in the fabrication process (up to $\pm 5 \mu \mathrm{m}$ ).

\section{Conclusions}

This paper presented detailed analytical modeling of capillary flow traversing a backward facing step in a microchannel with and without a top surface. The analytical model uses surface energy calculations to determine the capillary pressure as a function of the liquid-gas interface position in the microchannel. Furthermore, a geometric condition was set to check whether a liquid can flow spontaneously in a microchannel with a BFS.

The developed model was validated against numerical simulation results generated by two different simulation techniques; the Surface Evolver software employing surface energy minimization and ANSYS Fluent CFD simulation using volume of fluid method. Three different geometries and three different contact angles were used in the simulations. In general, the simulation results showed good agreement with the analytical model for both modeling methods. However, due to the assumption of 2D curvature of the meniscus surface in the $x-y$ plan only, the minimum value of the capillary pressure estimated from numerical simulation is more than the one determined by the analytical model especially for low contact angles and microchannel height to width aspect ratios. Hence, the analytical model works best at high contact angles $\left(\theta_{\mathrm{s}}>70^{\circ}\right)$ and high aspect ratios $\left(h_{1} / w>2\right)$.

Experimental tests were conducted to validate the spontaneous flow condition using test devices fabricated in silicon as both open and closed surface microchannels (covered by PDMS sheets for the closed surface case). Five different geometries were used for the experiments with aspect ratios ranging from 1.5 to 3.8 and contact angles ranging from $50^{\circ}$ to $85^{\circ}$ for silanized silicon oxide surfaces and from $93^{\circ}$ to $100^{\circ}$ for PDMS surfaces. Although some deviations were noted, the experimental results were in good agreement with the analytically derived condition. The developed model remains an enabling tool for designers of capillary microfluidic systems.

\section{Supplementary materials}

Supplementary material associated with this article can be found, in the online version, at doi:10.1016/j.ijmultiphaseflow.2018. 06.018.

\section{References}

Amin, T., Huda, M., Tulip, J., Jäger, W., 2012. Sidewall roughness control in deep reactive ion etch process for micromachined Si devices. In: Proc. 7th Int. Conf. Elect. Comput. Eng. (ICECE), pp. 82-85.

Berthier, J., Dubois, P., Clementz, P., Claustre, P., Peponnet, C., Fouillet, Y., 2007. Actuation potentials and capillary forces in electrowetting based microsystems. Sens. Actuators A 134, 471.

Brakke, K.A., 1992. The surface evolver. Exp. Math. 1, 141

Chakraborty, D., Chakraborty, S., 2010. Microfluidic transport and micro-scale flow physics: an overview. Microfluidics and Microfabrication. Springer.

Chen, J., Huang, P., Lin, M., 2008. Analysis and experiment of capillary valves for microfluidics on a rotating disk. Microfluid Nanofluid 4, 427-437.

Chibbaro, S., Costa, E., Dimitrov, D., Diotallevi, F., Milchev, A., Palmieri, D., Pontrelli, G., Succi, S., 2009. Capillary filling in microchannels with wall corrugations: a comparative study of the Concus-Finn criterion by continuum, kinetic, and atomistic approaches. Langmuir 25 21, 12653-12660.

Chin, C., Linder, V., Sia, S., 2012. Commercialization of microfluidic point-of-care diagnostic devices. Lab Chip 12, 2118-2134.

Cho, H., Kim, H., Kang, J., Kim, T., 2007. How the capillary burst microvalve works. J. Colloid Interface Sci. 306, 379-385. 
Geravis, L., Delamarche, E., 2009. Toward one-step point-of-care immunodiagnostics using capillary-driven microfluidics and PDMS substrates. Lab Chip 9, 3330-3337.

Gliere, A., Delattre, C., 2006. Modeling and fabrication of capillary stop valves for planar microfluidic systems. Sens. Actuators A 130, 601.

Hirt, C.W., Nichols, B.D., 1981. Volume of fluid (VOF) method for the dynamics of free boundaries. J. Comput. Phys. 39, 201.

Hitzbleck, M., Avrain, L., Smekens, V., Lovchik, R.D., Mertens, P., Delamarche, E., 2012. Capillary soft valves for microfluidics. Lab Chip 12, 1972-1978.

Hoang, D.A., van Steijn, V., Portela, L.M., Kreutzer, M.T., Kleijn, C.R., 2013. Benchmark numerical simulations of segmented two-phase flows in microchannels using the volume of fluid method. Comput. Fluids 86, 28.

Hoffman, R., 1983. A study of the advancing interface. I. Interface shape in liquid-gas systems. Colloid Interface Sci. 94 2, 470.

Junker, D., Schmid, H., Drechsler, U., Wolf, H., Wolf, M., Michel, B., Rooij, N., Delamarche, E., 2002. Autonomous microfluidic capillary system. Anal. Chem. 74, 6139-6144.

Kubiak, K.J., Wilson, M.C.T., Mathia, T.G., Carval, P., 2011. Wettability versus roughness of engineering surfaces. Wear 271 (3), 523-528.

Leu, T., Chang, P., 2004. Pressure barrier of capillary stop valves in micro sample separators. Sens. Actuators A 115, 508-515.

Majeed, B., Taher, A., Jones, B., Sbuncuoglu, D., 2015. Two step silicon microfluidics for capillary valve applications. In: International Symposium on Microelectronics, 2015, p. 555.

Man, P., Mastrangelo, C., Burns, M., Burke, D., 1998. Microfabricated capillaritydriven stop valve and sample injector. In: IEEE Micro Electro Mechanical Systems Workshop (MEM'98), pp. 45-50.

Melin, J., Roxhed, N., Gimenez, G., Griss, P., van der Wijngaart, W., Stemme, G., 2003. A liquid-triggered liquid microvalve. 12th International Conference on Solid State Sensors. Actuators, and Microsystems, June 8-12.
Safavieh, R., Juncker, D., 2013. Capillarics: pre-programmed, self-powered microfluidic circuits built from capillary elements. Lab Chip 13, 4180.

Saha, A.A., Mitra, S.K., 2009. Effect of dynamic contact angle in a volume of fluid (VOF) model for a microfluidic capillary flow. J. Colloid Interface Sci. 339, 461.

Vos, R., Rolin, C., Rip, J., Conard, T., Steylaerts, T., Cabanilles, M., Levrie, K., Jans, K., Stakenborg, T., 2018. Chemical vapor deposition of azidoalkylsilane monolayer films. Langmuir 34, 1400-1409.

Vulto, P., Podszun, S., Meyer, P., Hermann, C., Manzcd, A., Urban, G., 2011. Phaseguides: a paradigm shift in microfluidic priming and emptying. Lab Chip 11, 1596-1602

Weislogel, M., Lichter, S., 1998. Capillary flow in an interior corner. J. Fluid Mech. 373, 349-378.

Yildirim, E., Trietsch, S., Joore, J., van den Berg, A., Hankemeier, T., Vulto, P., 2014 Phaseguides as tunable passive microvalves for liquid routing in complex microfluidic networks. Lab Chip 14, 3334.

Yildirim, E., 2017. Modeling and Analysis of a Microfluidic Capillary Valve. Journal of Polytechnic 20 (2), 487-494.

Yuan, Y., Lee, T.R., 2013. Contact angle and wetting properties. Surface Science Techniques. Springer.

Zhang, L., Jones, B., Majeed, B., Nishiyama, Y., Okumura, Y., Stakenborg, T., 2018. Study on stair-step liquid triggered capillary valve for microfluidic systems. J. Micromech. Microeng. 28, 65005.

Zimmermann, M., Bentley, S., Schmid, H., Hunziker, P., Delamarche, E., 2005. Continuous flow in open microfluidics using controlled evaporation. Lab Chip 5, 1355

Zimmermann, M., Hunziker, P., Delamarche, E., 2008. Valves for autonomous capillary systems. Microfluid Nanofluid 5, 395-402. 